\title{
Intracellular cholesterol biosynthesis in enchondroma and chondrosarcoma
}

\author{
Hongyuan Zhang, ${ }^{1,2}$ Qingxia Wei, ${ }^{3,4}$ Hidetoshi Tsushima, ${ }^{5}$ Vijitha Puviindran, ${ }^{2}$ Yuning J. Tang, ${ }^{1,2}$ \\ Sinthu Pathmanapan, ${ }^{3}$ Raymond Poon, ${ }^{3}$ Eyal Ramu, ${ }^{3}$ Mushriq Al-Jazrawe, ${ }^{3}$ Jay Wunder, ${ }^{6}$ \\ and Benjamin A. Alman ${ }^{1,2}$ \\ 'Department of Cell Biology and 'Department of Orthopeadic Surgery, Duke University, Durham, North Carolina, USA. \\ ${ }^{3}$ Developmental and Stem Cell Biology, Hospital for Sick Children, Toronto, Ontario, Canada. ${ }^{4}$ First Affiliated Hospital of \\ Xi'an Jiaotong University, Xi'an, Shaanxi, China. ${ }^{5}$ Department of Orthopaedic Surgery, Kyushu University, Fukuoka, Japan. \\ ${ }^{6}$ Lunenfeld-Tanenbaum Research Institute, Mount Sinai Hospital, Toronto, Ontario, Canada.
}

Enchondroma and chondrosarcoma are the most common benign and malignant cartilaginous neoplasms. Mutations in isocitrate dehydrogenase 1 and 2 (IDH1/2) are present in the majority of these tumors. We performed RNA-seq analysis on chondrocytes from Col2a1Cre;/dh ${ }^{15 L /+}$ animals and found that genes implied in the cholesterol synthesis pathway were significantly upregulated in the mutant chondrocytes. We examined the phenotypic effect of inhibiting intracellular cholesterol biosynthesis on enchondroma formation by conditionally deleting sterol regulatory elementbinding protein cleavage-activating protein (SCAP), a protein activating intracellular cholesterol synthesis, in IDH1 mutant mice. We found fewer enchondromas in animals lacking SCAP. Furthermore, in chondrosarcomas, pharmacological inhibition of intracellular cholesterol synthesis substantially reduced chondrosarcoma cell viability in vitro and suppressed tumor growth in vivo. Taken together, these data suggest that intracellular cholesterol synthesis is a potential therapeutic target for enchondromas and chondrosarcomas.

Authorship note: $\mathrm{HZ}$ and QW contributed equally to this work.

Conflict of interest: The authors have declared that no conflict of interest exists.

Copyright: @ 2019, American Society for Clinical Investigation.

Submitted: January 8, 2019

Accepted: April 23, 2019

Published: June 6, 2019.

Reference information: /CI Insight. 2019;4(11):e127232. https://doi. org/10.1172/ji.insight.127232.

\section{Introduction}

Cartilage tumors as a group are the most common neoplasms affecting the bone (1). They include benign tumors, such as enchondromas or osteochondromas and malignant chondrosarcomas (2). Enchondromas arise from dysregulated growth plate chondrocytes that fail to undergo terminal differentiation and remain at the end of the bone (2, 3). They can occur within the medullary bone as a single lesion or as multiple lesions (enchondromatosis) in conditions termed Ollier disease and Maffucci syndrome (2). Multiple enchondromas in Maffucci syndrome are associated with up to a $50 \%$ chance of these lesions undergoing malignant transformation to form chondrosarcomas $(3,4)$. Chondrosarcoma is the second most common malignant primary bone tumor and is resistant to chemotherapy and radiation therapy (5). Somatic mutations in the isocitrate dehydrogenase 1 and 2 (IDH1 and $I D H 2$ ) genes are identified in the majority of enchondromas and about half of chondrosarcomas (6-8). Expression of a mutant $I d h 1$ in chondrocytes is sufficient to initiate enchondroma formation in mice by disrupting chondrocyte differentiation (9). When the mutant $I d h 1$ was expressed in chondrocytes during development, the mice displayed severe defects in chondrocytes differentiation and could not survive after birth. When the mutant $I d h 1$ was expressed in chondrocytes postnatally, the mice developed enchondromatosis (9). D-2-hydroxyglutarate (D-2HG), which is commonly believed to be an "oncometabolite," is produced from mutant IDH at high levels. However, inhibition of D-2HG production did not alter cell viability of chondrosarcomas, suggesting mutant IDH may promote tumor growth via other mechanisms (4).

Intracellular cholesterol biosynthesis plays a crucial role in chondrocyte development. This process is regulated by the protein sterol regulatory element-binding protein cleavage-activating protein (SCAP). When intracellular cholesterol level is low, SCAP cleaves and activates the transcription factors known as SREBPs (ref. 10). After cleavage, SREBPs are further processed and translocate to the nucleus to activate genes responsible for cholesterol biosynthesis (10). Genetic deletion of Scap in chondrocytes affected their differentiation and viability (11). In addition, pharmacological inhibition of cholesterol synthesis by statin drugs caused reduced endochondral bone growth and decreased height of the growth plate (12). Furthermore, statin drugs rescued chondrocyte differentiation and bone length in models of achondroplasia (13). 
Deregulation of cholesterol homeostasis has been identified in multiple cancer types and is believed to be an important contributing factor to cancer progression (14). Upregulation of the cholesterol synthesis pathway is associated with decreased patient survival in sarcoma, acute myeloid leukemia, and melanoma (14). Serum and intracellular cholesterol levels may not always be directly related, and studies suggest that intracellular cholesterol homeostasis may have a more important role in cancers than the serum cholesterol (14). Despite this information, the role of cholesterol in cartilage tumors is not known.

In this study, we investigated genes that were differentially expressed in IdhI mutant chondrocytes and found that genes that are activated in cholesterol biosynthesis were upregulated. To determine the role of cholesterol biosynthesis in enchondroma and chondrosarcoma, we genetically and pharmacologically modulated this pathway and found that inhibition of cholesterol synthesis inhibited cartilage tumor formation and growth.

\section{Results}

Genes that activate intracellular cholesterol synthesis are upregulated in Idh1-KI chondrocytes. IDH1-R132Q was identified in a patient's chondrosarcoma tumor (9). We generated a mouse expressing IDH1- R132Q in chondrocytes by crossing a mouse expressing a conditional Idh1-R132Q knockin allele (15) with a mouse expressing Cre recombinase driven by regulatory elements of the type 2 collagen gene (Col2a1-Cre) (16). As reported previously, these animals displayed severe defects in chondrocyte differentiation during development and were rarely found alive after birth (9). They exhibited defects in chondrocyte terminal differentiation. Mice in which the conditional allele was activated postnatally in chondrocytes developed multiple enchondroma-like lesions in the metaphysis of bone (9). To determine how Idh1 mutation regulates chondrocyte differentiation, we performed RNA-seq analysis on primary sternal chondrocytes isolated from Col2a1Cre;IdhILSL/+ $(I d h 1-\mathrm{KI})$ animals and Idh1 $1^{L S L /+}$, Col2a1Cre littermate controls.

RNA-seq revealed distinct expression profiles between Idh1-KI animals versus the 2 control groups (GSE123130; Figure 1A). Gene set enrichment analysis (GSEA) showed that genes regulating the cholesterol biosynthesis pathway were markedly upregulated in Idh1-KI chondrocytes (Figure 1B). As an example, Srebf2, the gene encoding for the transcription factor that activates cholesterol synthesis, was significantly upregulated (Figure 1C). Enzymes in the mevalonate pathway, which is an essential process for cholesterol synthesis, were consistently upregulated in Idh1-KI chondrocytes (Figure 1C). We confirmed the upregulation of genes in the pathway, such as $H m g c r$, the rate-limiting enzyme in the mevalonate pathway, by qPCR (Figure 1D). To determine changes in cholesterol levels, we performed filipin staining and found significantly higher levels of filipin staining intensity in Idh1-KI chondrocytes compared with chondrocytes from control animals (Figure 2, A and B). These data consistently suggest cholesterol biosynthesis is activated in chondrocytes expressing mutant Idh1.

Deleting Scap postnatally did not alter the growth plate phenotype. SCAP activates the intracellular cholesterol synthesis pathway by cleaving and activating transcription factors known as SREBPs (10). We examined the role of intracellular cholesterol in chondrocytes by genetically deleting Scap. We generated Col2a1Cre ${ }^{\mathrm{ERT} 2} ;$ Scap ${ }^{f / f l}$ (Scap-CKO) mice in which Scap was conditionally deleted in Col2a1-expressing cells upon tamoxifen administration at 4 weeks. Recombination was confirmed by PCR. The phenotype of the growth plate cartilage in Scap-CKO mice was examined at 6 months of age. Histological evaluation did not reveal an obvious phenotype in the growth plate cartilage of Scap-CKO mice (Supplemental Figure 1A; supplemental material available online with this article; https://doi.org/10.1172/jci.insight.127232DS1). The presence of early-stage chondrocytes in the growth plate was not altered, as shown by immunohistochemistry of SOX9 (Supplemental Figure 1B). Hypertrophic differentiation of growth plate chondrocytes was not affected by Scap deletion, as shown by immunohistochemistry of type X collagen (Supplemental Figure 1C). Deletion of Scap did not cause abnormal cell proliferation, as shown by immunohistochemistry of Ki67 (Supplemental Figure 1D). Together, these data suggest growth plate chondrocytes in adult mice were not affected by Scap deletion under physiological condition.

Deleting Scap reduced enchondroma-like lesion formation in Idh1-KI animals. To determine if intracellular cholesterol synthesis is important in enchondroma formation, we studied it in mice expressing mutant Idh1 (Idh1-KI). We generated Col2a1Cre ${ }^{\text {ERT2; Scap }}{ }^{f l f l} ; I d h 1^{L S L /+}$ mice in which Scap deletion and mutant Idh1 expression were simultaneously induced by tamoxifen administration in Col2a1-expressing cells. Enchondroma-like lesions still formed in the absence of Scap (Figure 3A). However, quantification showed reduced number and size of enchondroma-like lesions in Idh1-KI animals lacking Scap (Figure 3, B-D). These data show that inhibiting cholesterol synthesis reduced enchondroma-like lesion formation in Idh1-KI mice. 

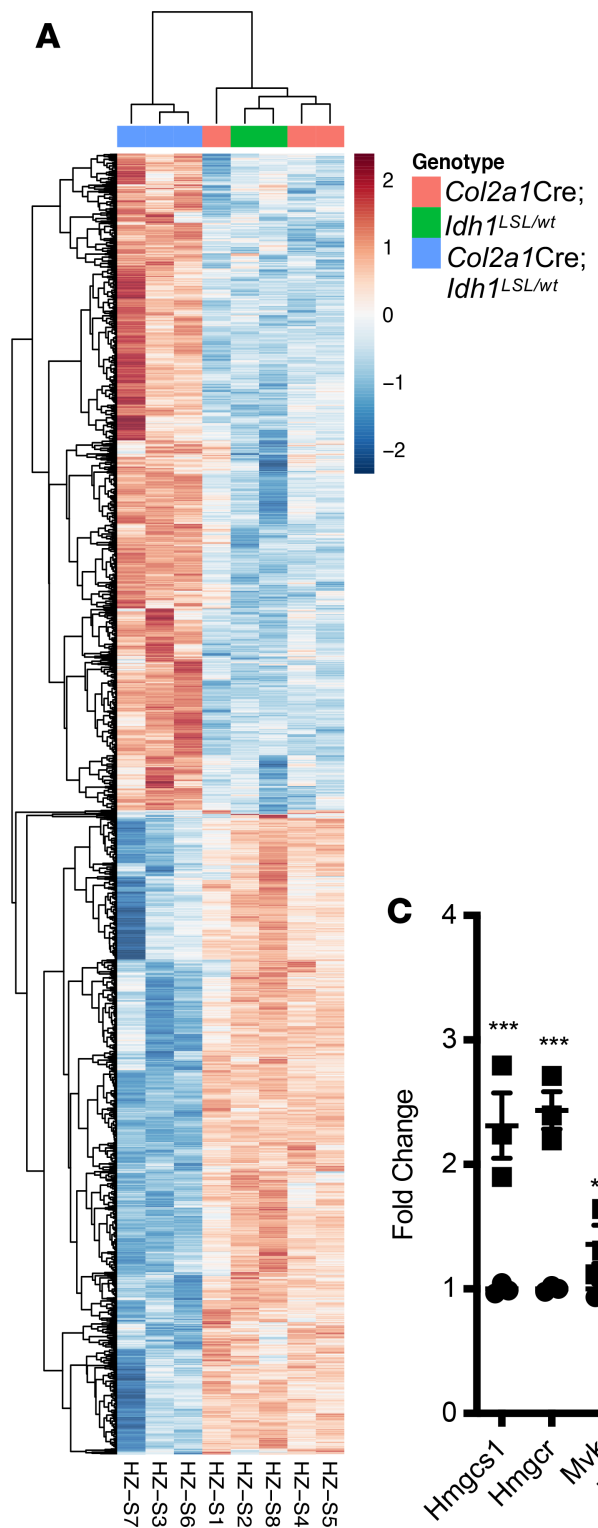

\section{B}
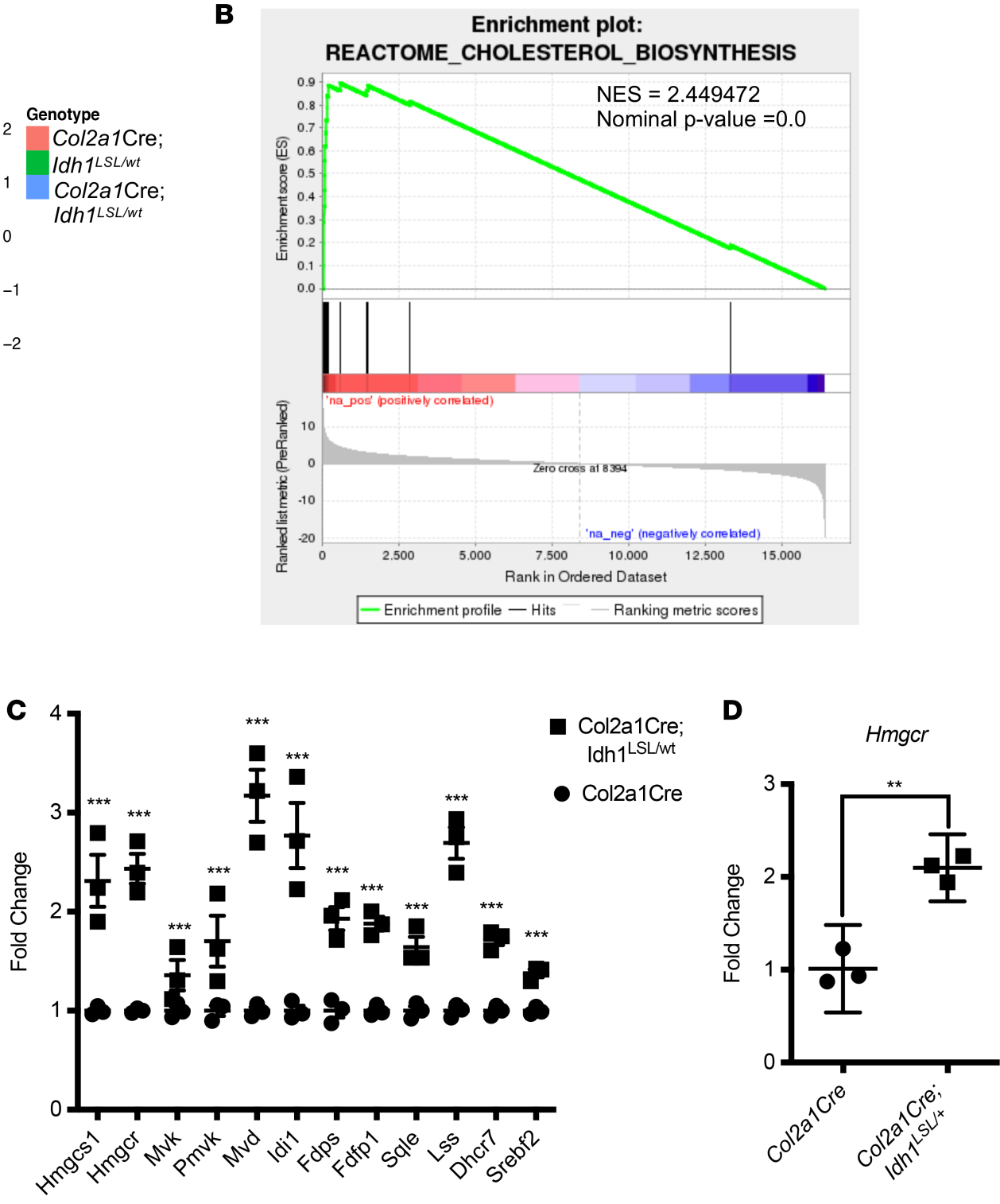

Figure 1. Cholesterol synthesis was upregulated in IDH1 ${ }^{R 1320}$ chondrocytes. (A) Heatmap of RNA-seq on sternal chondrocytes from Col2a1Cre; $/ d h 7^{L S L /+}(n=3)$, Idh $1^{L L L /+}(n=2)$, and Col2a1Cre $(n=3)$ mice at E18.5. Littermates were used for the analysis. (B) Gene set enrichment analysis for the cholesterol biosynthesis pathway. (C) Relative fold change of gene expression in the cholesterol synthesis pathway $(n=3)$. ${ }^{* *} P<0.001$. The false discovery rate was calculated to control for multiple hypothesis testing. (D) qPCR of Hmgcr of sternal chondrocytes isolated from Col2a1Cre and Col2a1Cre;/dh1LLL/+ animals at E18.5 $(n=3)$. ${ }^{* *} P<0.01$, unpaired, 2 -tailed Student's $t$ test. Mean $\pm 95 \%$ confidence intervals are shown.

Pharmacological inhibition of cholesterol synthesis reduced chondrosarcoma growth in vitro and in vivo. Enchondromas can progress into malignant chondrosarcomas. To examine the role of intracellular cholesterol in chondrosarcoma, we first evaluated cholesterol levels in chondrosarcomas with or without IDH mutations. We measured cholesterol levels in cryopreserved primary chondrosarcoma tissues from patients with wildtype IDH enzymes or mutant IDH1. Chondrosarcoma tumors with mutant IDH1 had significantly higher cholesterol levels than the tumors with wild-type IDH1/2 (Figure 4A). To modulate cholesterol synthesis in chondrosarcoma, we treated primary chondrosarcoma cells derived from 5 different patients with lovastatin, which inhibits HMG-CoA reductase, the rate-limiting enzyme in the cholesterol biosynthetic pathway (10). One of the five patient samples was wild-type for IDH1/2 and the other 4 samples had IDHI mutation. Treatment of chondrosarcoma cells with lovastatin caused a substantial reduction of cell viability in vitro (Figure 4B). We also treated chondrosarcoma explants that were derived from the 5 patients with lovastatin in vitro. The proliferation rate in chondrosarcoma explants was significantly reduced, 
A

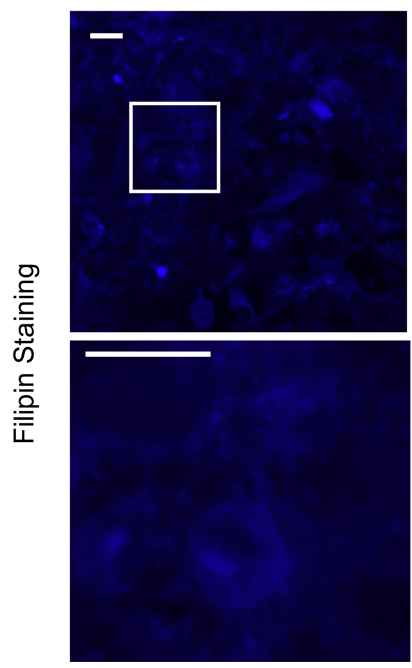

Col2a1Cre; Idh 1 LSL/+

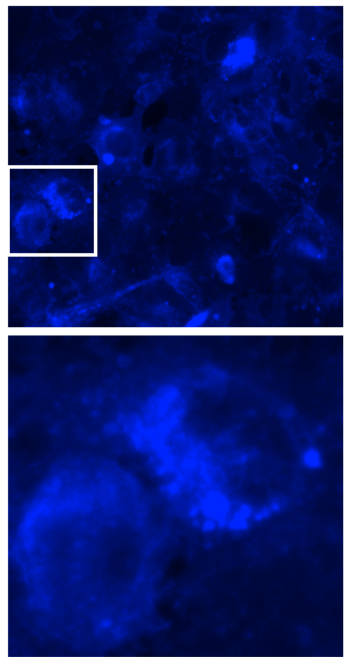

B

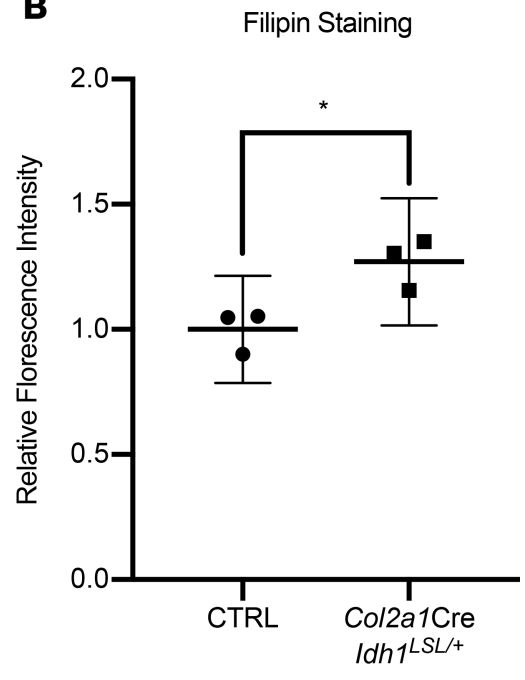

Figure 2. Cholesterol levels were higher in Idh1-KI chondrocytes. (A) Representative filipin staining of sternal chondrocytes isolated from Col2a1Cre and Col2a1Cre; $/ d h 1^{L L L /+}$ mice at E18.5. (B) Quantification of the filipin staining intensity. The control group included 1 Col2a1Cre animal and 2 wild-type animals. Littermates were used for the analysis. Scale bars: $25 \mu \mathrm{m} . n=3$. ${ }^{*} P<0.05$, unpaired, 2-tailed Student's $t$ test. Mean $\pm 95 \%$ confidence intervals are shown.

as shown by immunohistochemistry of Ki67 (Figure 4, C and D). Chondrosarcoma thus joins other tumor types that respond to cholesterol synthesis inhibition with reduced cell viability (17).

To examine the effects of lovastatin on chondrosarcoma growth in vivo, we generated patient-derived xenografts of chondrosarcomas in NOD/SCID- $\gamma$ (NSG) mice as previously described (18). We examined tumor growth in xenografts established from 5 different patient tumors. Each patient-derived chondrosarcoma was xenografted to 10 animals. Five animals were treated with vehicle control and five animals were treated with lovastatin. The treatment started 3-4 weeks after injection of tumor cells, a time when the tumor became palpable. For each individual human xenograft, control and lovastatin treatments were started at the same time. In sample 1, IDH1 and $I D H 2$ were wild-type. Samples 2, 3, 4, and 5 harbored mutations in IDH1. After 4 weeks of treatment, tumor weight was measured. Because there was a high degree of variation among tumor weights from different patients, we normalized the tumor weight of each tumor to the average tumor weight of the control group derived from the same patient. Lovastatin treatment caused an average of 30\% reduction of tumor weight (Figure 5A). Proliferation rate of the xenografted chondrosarcoma tumors was examined by BrdU staining. The relative proliferation rate was determined by normalizing the percentage of BrdU-positive cells of each tumor to the average percentage of BrdU-positive cells of the control group derived from the same patient. Lovastatin reduced proliferation in all 5 patient-derived tumor xenografts (Figure 5B and Supplemental Figure 2A). Apoptosis was examined by immunohistochemistry of cleaved caspase- 3 . The relative apoptosis rate was determined by normalizing the percentage of cleaved caspase-3-positive cells of each tumor to the average percentage of cleaved caspase-3 positive cells of the control group derived from the same patient. Lovastatin treatment increased apoptosis of chondrosarcoma cells in vivo (Figure 5C). For 4 of 5 patient-derived xenografts, the average apoptosis rates were increased by lovastatin treatment. For sample 1 (wild-type $I D H$ ), the percentage of cleaved caspase-3-positive cells was not altered by lovastatin treatment (Supplemental Figure 2B), suggesting apoptosis in wild-type IDH chondrosarcomas might not be affected by inhibition of intracellular cholesterol biosynthesis in vivo.

\section{Discussion}

$I D H 1 / 2$ are the most commonly mutated genes in enchondroma and chondrosarcoma. Mutation in Idh1 in chondrocytes causes defects in their terminal differentiation and results in the formation of enchondroma-like lesions. In this study, we found that $I d h 1$ mutation in chondrocytes increased intracellular cholesterol biosynthesis, which was important in regulating the number and size of enchondroma-like lesions, the viability of chondrosarcoma cells, and the tumor growth of chondrosarcomas. 
A
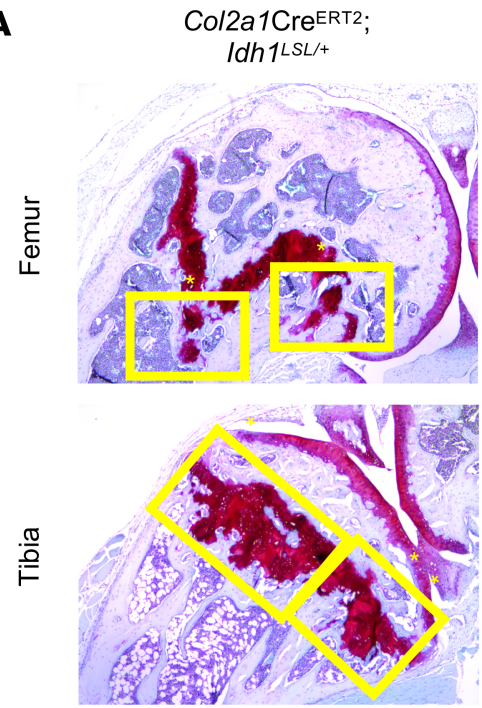

B

Number of enchondroma-like lesions

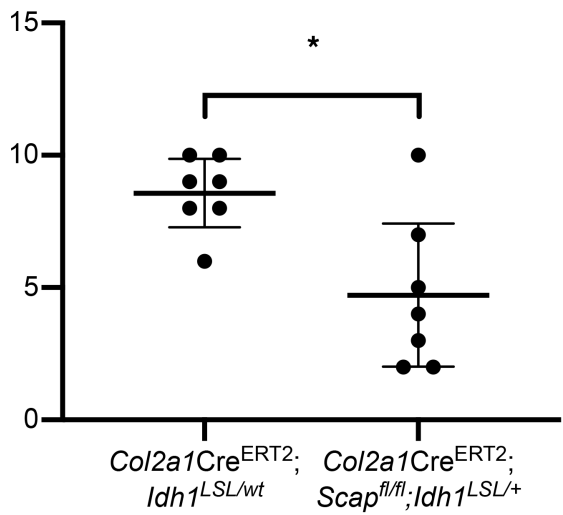

D
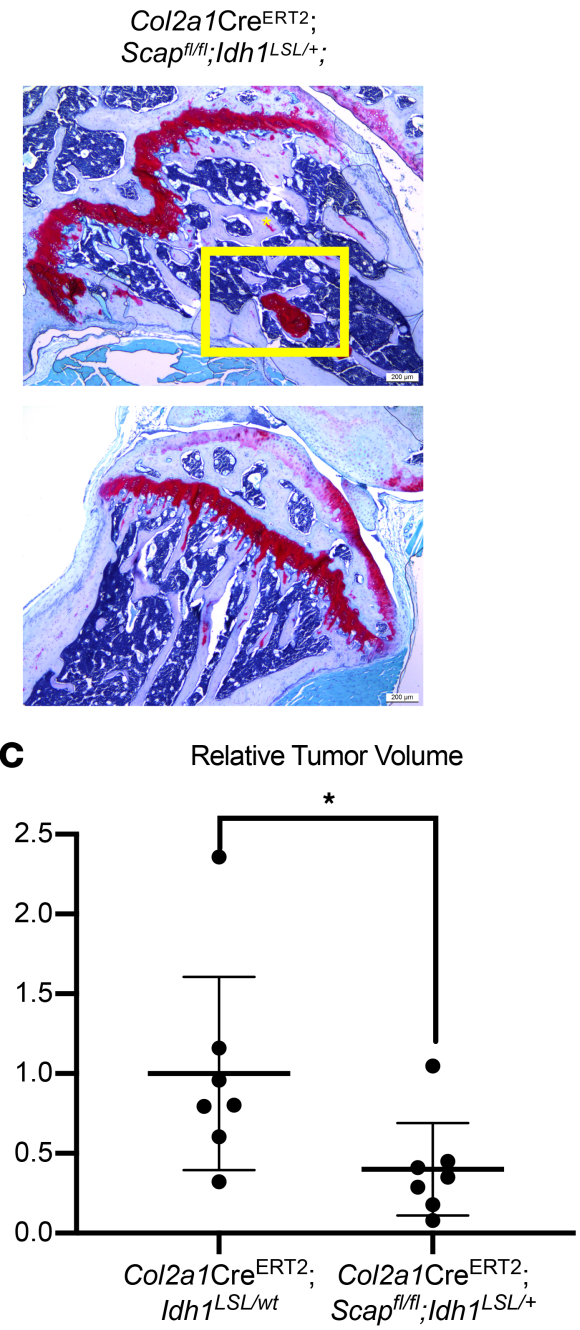

Col2a1CreERT2;

Scap fl/fl; Idh1 LSL/+
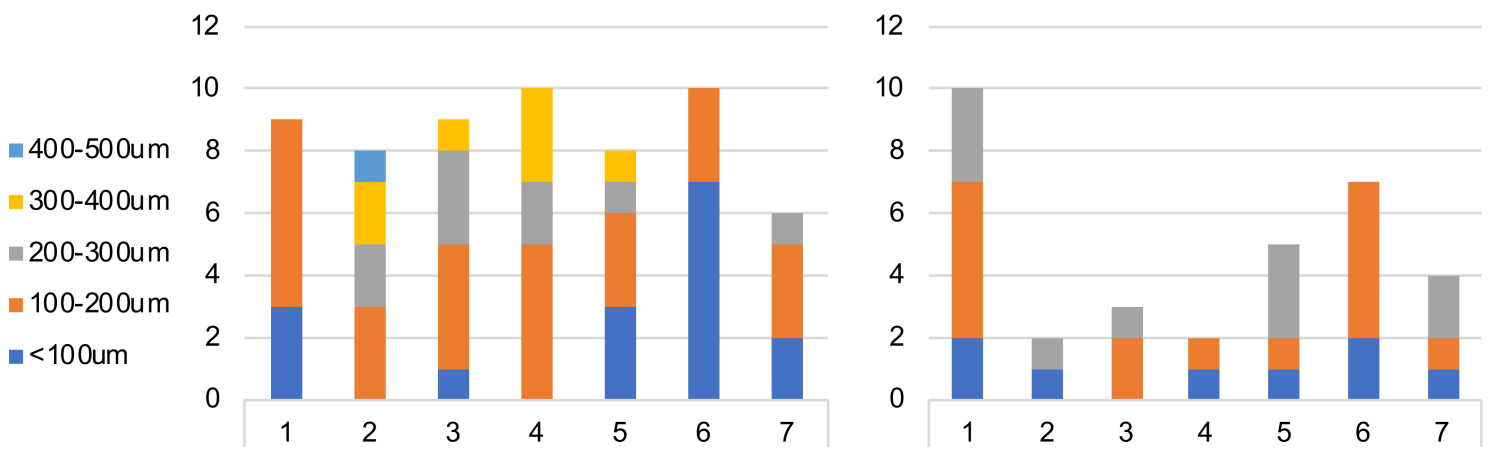

Figure 3. Deleting Scap reduced enchondroma-like lesions in Idh1-KI mice. (A) Representative Safranin 0 staining of Col2alCre ${ }^{\mathrm{ERT}}$;Idh1LLL/+ and Col2a1Cre ${ }^{\mathrm{ERT}}$;Scap ${ }^{f l / f} ; \mathrm{Idh} 7^{\mathrm{LSL} /+}$ mice. (B) Number of enchondroma-like lesions. (C) Relative tumor volume of enchondroma-like lesions. (D) Distribution of the width of enchondroma-like lesions. Scale bars: $200 \mu \mathrm{m}$. Each data point represents 1 animal. $n=7 .{ }^{*} P<0.05$, unpaired, 2 -tailed Student's $t$ test. Mean $\pm 95 \%$ confidence intervals are shown.

At the time of analysis, some enchondroma-like lesions had grown separate from the growth plates and

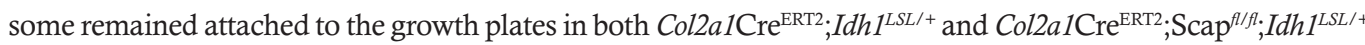
animals. In human patients with enchondromatosis, the tumors are attached to the growth plates initially and stay in this location after the growth plates close. Because the growth plates never close in mice, the enchondroma-like lesions that formed later during growth would remain attached to the growth plate throughout life. 

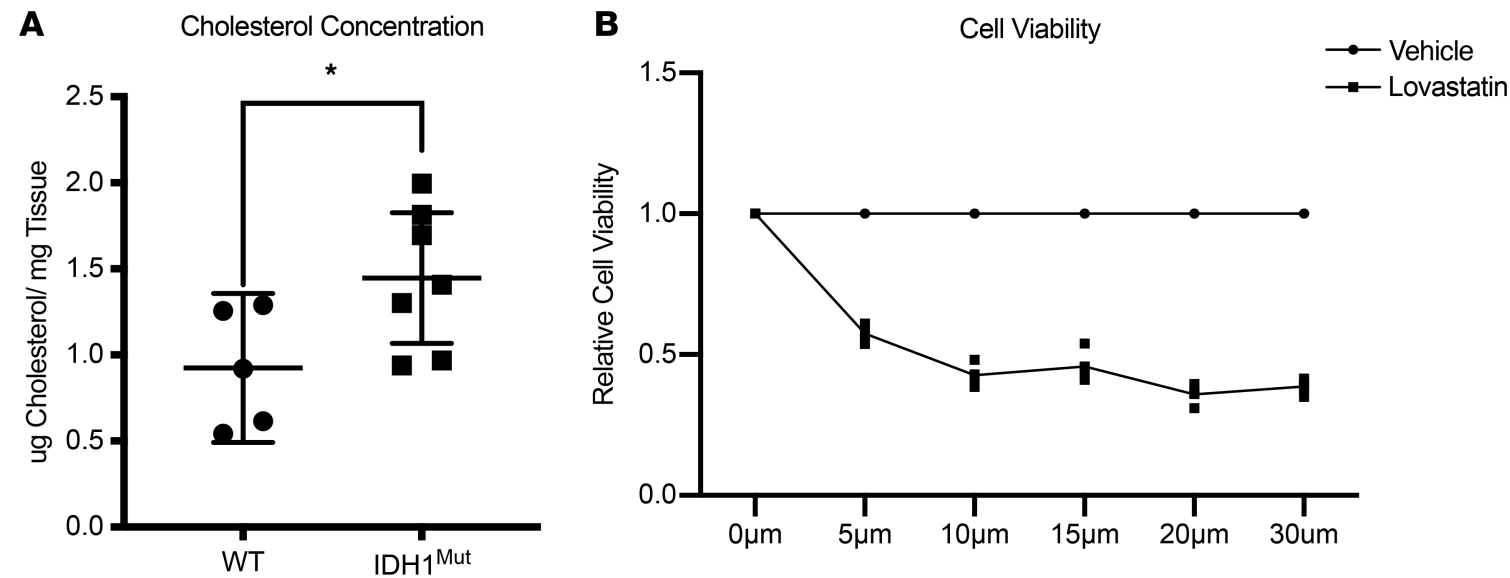

C

Control

Lovastatin
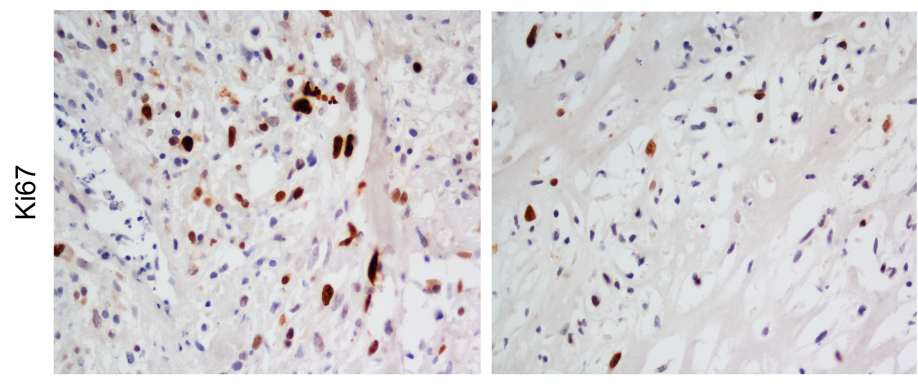

D

Proliferation

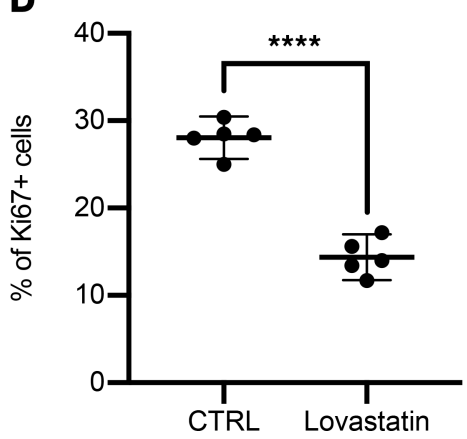

Figure 4. Lovastatin inhibited chondrosarcoma viability in vitro. (A) Cholesterol concentration in primary chondrosarcoma tumors with wild-type IDH1/2 $(n=5)$ or mutant $I D H 1(n=7)$. (B) Relative cell viability of chondrosarcoma cells after lovastatin treatment for 48 hours at indicated concentrations in vitro $(n=5)$. (C) Representative immunohistochemistry for Ki67 on chondrosarcoma explants treated with vehicle control or lovastatin (original magnification, $\times 200)$. (D) Quantification of the percentage of Ki67-positive cells in $\mathbf{C}(n=5)$. For $\mathbf{A}, \mathbf{B}$, and $\mathbf{D}$, each data point represents a chondrosarcoma from an individual patient. ${ }^{*} P<0.05,{ }^{* * *} P<0.0001$, unpaired, 2 -tailed Student's $t$ test. Mean $\pm 95 \%$ confidence intervals are shown.

Intracellular cholesterol regulates chondrocyte differentiation under various conditions. During development, genetic deletion of regulators of cholesterol synthesis disrupted the hypertrophic differentiation of chondrocytes and resulted in shortening limbs by altering hedgehog signaling (11). In an achondroplasia model with FGFR3 gain-of-function mutation, statins restored chondrocyte differentiation and caused significant bone recovery, suggesting that cholesterol could regulate chondrocyte differentiation via crosstalk with FGF signaling (13). In addition, it was reported abnormal cholesterol levels were associated with the development of osteoarthritis (19).

The mechanisms of which cholesterol biosynthesis regulates tumor growth of enchondroma and chondrosarcoma are not fully elucidated. However, deregulation of cholesterol biosynthesis is known to contribute to cancer growth of other cancer types via multiple mechanisms (14). In prostate cancer, upregulation of cholesterol via PI3K/AKT/mTOR signaling promoted cancer aggressiveness and bone metastases (14, 20). In hepatocellular carcinoma and colon cancer, increased mitochondrial cholesterol content induced resistance to apoptosis $(14,21)$. Statins have been used alone or combined with other drugs for multiple types of cancers over the past few decades. They have shown to be especially effective in the treatment of mesenchymal-like cancer cells $(14,22)$.

Previous studies have showed that expression of wild-type and mutant Idh 1 is activated by SREBP1 and SREBP2 (encoded by Srebf1 and Srebf2) in livers as well as multiple cancer cell lines (23-25), and our study demonstrated that mutant Idh1 upregulated Srebf2. Since expression of mutant IDH1 has been previously shown to affect histone modification and DNA methylation $(26,27)$, it may be possible that expression of Srebf2 in Idh1 mutant chondrocytes could be altered via epigenetic changes. Mutant IDH1 is also known to cause metabolic reprogramming, such as changing redox states (28), which could also be a potential mechanism that regulates intracellular cholesterol biosynthesis. 
A

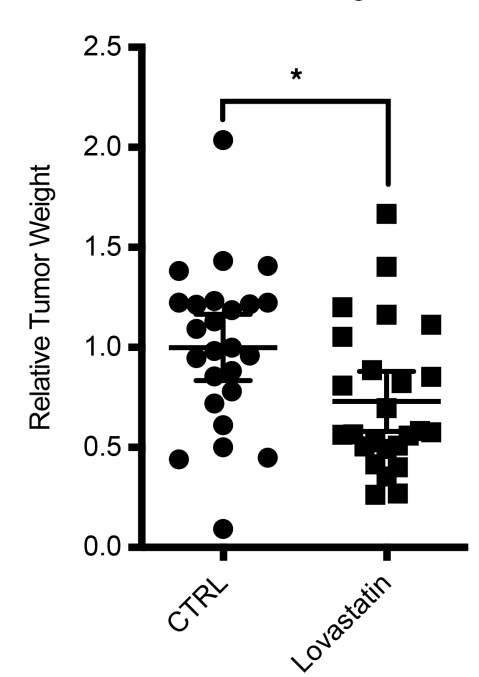

C

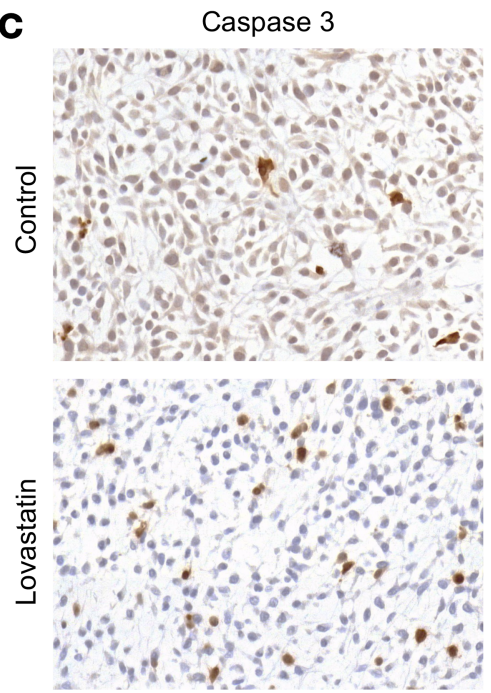

B

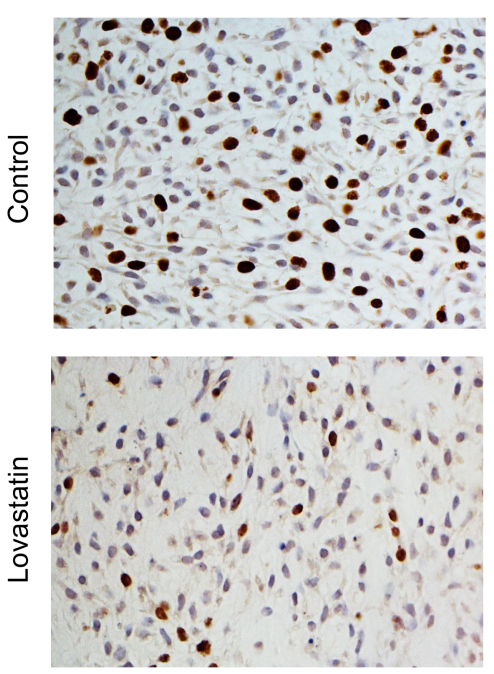

Proliferation

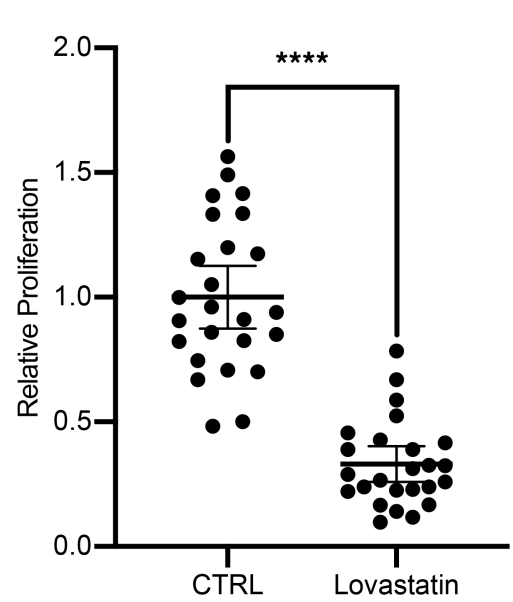

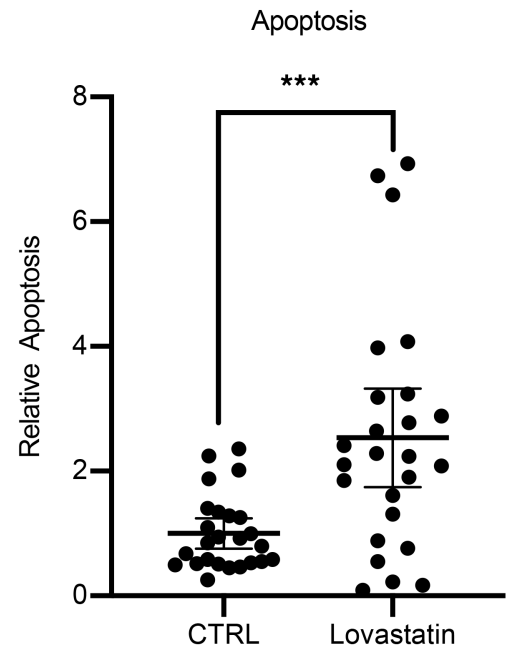

Figure 5. Lovastatin inhibited chondrosarcoma growth in vivo. (A) Relative tumor weight of chondrosarcoma xenografts after vehicle or lovastatin treatment. (B) Representative images of BrdU staining on xenografted tumors and quantification of BrdU-positive cells in relative values (original magnification, $\times 200$ ). (C) Immunohistochemistry of cleaved caspase-3 on xenografted tumors and quantification of cleaved caspase-3-positive cells in relative values (original magnification, $\times 200$ ). Each data point represents the relative tumor weight, relative percentage of BrdU-positive cells, or relative percentage of cleaved caspase-3-positive cells of each xenograft tumor. $n=25 .{ }^{*} P<0.05,{ }^{* * *} P<0.001,{ }^{* * *} P<0.0001$, unpaired, 2-tailed Student's $t$ test. Mean $\pm 95 \%$ confidence intervals are shown

In conclusion, our study demonstrated that intracellular cholesterol synthesis was upregulated in Idh1-KI chondrocytes. Genetic and pharmacological inhibition of intracellular cholesterol synthesis suppressed growth in enchondroma-like lesions and chondrosarcoma. Our study identified the cholesterol synthesis pathway as a potential therapeutic target in enchondroma and chondrosarcoma, which currently do not have any universally effective therapeutics. As $I D H 1 / 2$ are also frequently mutated in other cancers such as glioblastoma and acute myeloid leukemia, our data suggested statin drugs could be a potential therapeutic for these cancers as well.

\section{Methods}

Animals. Mice used in this study include Scap ${ }^{f l f l}$ mice (29), Idh $1^{L S L /+}$ mice (15), Col2a1Cre mice (16), Col2a1Cre ${ }^{\mathrm{ERT} 2}$ mice (30), and NSG mice (31). All the mice other than NSG mice were on BL6 background. Idh $1^{\text {LSL/+ }}$ mice bear an R132Q mutation rather than an $\mathrm{R} 132 \mathrm{H}$ mutation as previously clarified (9). Adult growth plate and enchondroma-like phenotypes were analyzed on 6-month-old mice. Tamoxifen was administered daily via intraperitoneal injection for 10 days at $100 \mathrm{mg} / \mathrm{kg}$ body weight/day at 4 weeks of age. Hind limbs were harvested for histological analysis. 
Xenograft. Primary chondrosarcoma cells derived from 5 different patients were used in the study. Chondrosarcoma cells derived from each patient were xenografted onto 10 animals: 5 animals for lovastatin treatment and 5 animals for vehicle treatment. One million chondrosarcoma cells were subcutaneously injected into each NSG mouse. Lovastatin and vehicle control treatment started 3-4 weeks after injection, a time when the tumor became palpable. For each individual human xenograft, the controls and treatments were started at the same time following implantation. The mice were treated with lovastatin $(4.5 \mathrm{mg} / \mathrm{kg} / \mathrm{d})$ or vehicle $(100 \%$ ethanol) for 4 weeks. $5 \mathrm{mg} / \mathrm{kg}$ bodyweight of BrdU was administered via intravenous infusion 4 hours before sacrificing the mice. Tumors were harvested, weighted, and processed for immunohistochemistry.

Histological analysis. For tissue processing, hind limbs were fixed in 10\% neutral buffered formalin for 3 days, decalcified with 14\% EDTA for 2 weeks at room temperature, and embedded in paraffin. Xenografted tumors were fixed in 4\% PFA overnight. Tissues were sectioned at $5 \mu \mathrm{m}$ and used for Safranin O staining and immunohistochemistry. For type $\mathrm{X}$ collagen, antigen retrieval was performed by citrate buffer incubation $\left(85^{\circ} \mathrm{C}, 15 \mathrm{~min}\right)$ and hyaluronidase digestions $\left(10 \mathrm{mg} / \mathrm{ml}, 37^{\circ} \mathrm{C}, 30 \mathrm{~min}\right)$. For BrdU, Ki67, and cleaved caspase-3, antigen retrieval was performed by boiling the slides in pressure cooker for 3 minutes in 10 $\mathrm{mM}$ citrate buffer $(\mathrm{pH}=6.0)$. Endogenous peroxidase was blocked by $3 \% \mathrm{H}_{2} \mathrm{O}_{2} /$ methanol $(\mathrm{v} / \mathrm{v})(10 \mathrm{~min}$, room temperature) and the Dako Dual Endogenous Enzyme Block For Autostainer (Agilent Technologies, S2003) (30 min, room temperature). The specimen was blocked with $2 \%$ horse serum (30 min, room temperature) and incubated with antibodies for Col X (1:500, Thermo Fisher Scientific, X53), Ki67 (DAKO, MIB-1),BrdU (1:50, CST Bu20a), and cleaved caspase-3 (1:200, Cell Signaling, Asp175) at $4^{\circ} \mathrm{C}$, overnight.

Quantification of enchondroma-like lesions. Enchondroma-like lesions were firstly detected by Safranin $\mathrm{O}$ staining. We stained 1 slide ( 2 sections, $10 \mu \mathrm{m})$ in every 10 slides $(100 \mu \mathrm{m})$ to identify enchondroma-like lesions. We then examined every section of each bone under the microscope to determine the exact number of sections each enchondroma-like lesion spans and identify any lesions that were missed by Safranin $\mathrm{O}$ staining. In this way, we examined these lesions continuously. Each section was $5 \mu \mathrm{m}$ thick. The width of each lesion was determined by the number of sections the lesion spanned. For every Safranin O-stained section, we manually outlined each lesion and measured lesion area using the image processing software Fiji ImageJ. We estimated tumor volume of each animal by adding up the lesion areas of every Safranin O-stained section. Relative tumor volume was determined by normalizing the tumor volume of each animal to the average tumor volume of $C o l 2 a 1 \mathrm{Cre} e^{\text {ERT2 }} ; I d h 1^{\text {LSL } /+}$ animals.

Isolation of primary sternal chondrocytes. Costal chondrocytes were isolated from E18.5 embryos. Mouse sterna and ribs were digested by Pronase (Roche) $(2 \mathrm{mg} / \mathrm{ml})$ for 30 minutes with constant agitation at $37^{\circ} \mathrm{C}$, washed by PBS, and then digested by Collagenase IV (Worthington) $\left(3 \mathrm{mg} / \mathrm{ml}\right.$ ) for 1 hour in $37^{\circ} \mathrm{C}$ humidified chamber, washed by PBS, digested by Collagenase IV $(0.5 \mathrm{mg} / \mathrm{ml})$ for 16 hour in $37^{\circ} \mathrm{C}$ humidified chamber, and filtered using $45-\mu \mathrm{m}$ cell strainer.

Culture of primary sternal chondrocytes. Costal chondrocytes from $I d h 1^{L S L /+}$ mice were cultured in DMEM with 10\% FBS and 1\% Penicillin/Streptomycin (Thermo Fisher Scientific).

Filipin staining and quantification. Filipin staining (Abcam, ab133116) was performed on sternal chondrocytes according to the manufacturer's instructions. Primary sternal chondrocytes were isolated from E18.5 mouse embryos from the same litter. We quantified the fluorescent intensity of the filipin staining using the image processing software Fiji ImageJ. Outlines of cells were drawn manually. Mean fluorescence intensities of chondrocytes isolated from control and Idh1-KI animals were measured. Ten fields of each sample were used for measurement, and the average of the ten measurements was used as the intensity of that sample. The relative intensity was given as a ratio of the intensity of each animal normalized to the average intensity of control animals.

$R N A$-seq. RNA-seq analysis was performed on sternal chondrocytes from Col2a1Cre, Idh $1^{L S L /+}$, Col2a1Cre;Idh1 $1^{L S L /+}$ animals at E18.5. RNA was extracted using the RNeasy Mini Kit (QIAGEN). Extracted total RNA quality and concentration was assessed on a 2100 Bioanalyzer (Agilent Technologies) and Qubit 2.0 (Thermo Fisher Scientific), respectively. Only extracts with RNA integrity number greater than 7 were processed for sequencing. RNA-seq libraries were prepared using the commercially available KAPA Stranded mRNA-Seq Kit. In brief, mRNA transcripts were first captured using magnetic oligo-dT beads, fragmented using heat and magnesium, and reverse transcribed using random priming. During the second-strand synthesis, the cDNA/ RNA hybrid was converted into to double-stranded cDNA (dscDNA) and dUTP incorporated into the second cDNA strand, effectively marking the second strand. Illumina sequencing adapters were then ligated to the 
dscDNA fragments and amplified to produce the final RNA-seq library. The strand marked with dUTP was not amplified, allowing strand-specificity sequencing. Libraries were indexed using a 6-base pairs index, allowing for multiple libraries to be pooled and sequenced on the same sequencing lane on a HiSeq 4000 Illumina sequencing platform. Before pooling and sequencing, fragment length distribution and library quality was first assessed on a 2100 Bioanalyzer using the High Sensitivity DNA Kit (Agilent Technologies). All libraries were then pooled in equimolar ratio and sequenced. Multiplexing 8 libraries on one lane of an Illumina HiSeq 4000 flow cell yielded about 40 million 50 bp single end sequences per sample. Once generated, sequence data were demultiplexed and Fastq files generated using Bcl2Fastq conversion software provided by Illumina.

$R N A$-seq analysis. RNA-seq data were processed using the TrimGalore toolkit, which employs Cutadapt (32) to trim low-quality bases and Illumina sequencing adapters from the 3 ' end of the reads. Only reads that were $20 \mathrm{nt}$ or longer after trimming were kept for further analysis. Reads were mapped to the GRCm38v75 version of the mouse genome and transcriptome (33) using the STAR RNA-seq alignment tool (34). Reads were kept for subsequent analysis if they mapped to a single genomic location. Gene counts were compiled using the HTSeq tool. Only genes that had at least 10 reads in any given library were used in subsequent analysis. Normalization and differential expression was carried out using the DESeq2 (35) Bioconductor (36) package with the $\mathrm{R}$ statistical programming environment. The false discovery rate was calculated to control for multiple hypothesis testing. Gene set enrichment analysis was performed to identify differentially regulated pathways and gene ontology terms for each of the comparisons performed (37).

Chondrosarcoma cell and explant culture. Chondrosarcoma cells and/or xenograft explants were cultured in MEM- $\alpha$ with 10\% FBS and 1\% Penicillin/Streptomycin. Cells/explants were treated with $20 \mu \mathrm{M}$ lovastatin and vehicle ( $100 \%$ ethanol) for 48 hours. Cell viability was measured by SRB cell cytotoxicity assay.

Measurement of cholesterol levels. We measured cholesterol levels from cryopreserved chondrosarcoma tissues using the cholesterol/cholesteryl ester assay kit (Abcam, ab65359) according to the manufacturer's instruction. $10 \mathrm{mg}$ tissue from each chondrosarcoma was used for the measurement.

Statistics. For RNA-seq analysis, the false discovery rate was calculated to control for multiple hypothesis testing. Other statistical analyses were performed using unpaired, 2-tailed Student's t test. Statistical significance was determined as $P<0.05$. All data were presented as mean $\pm 95 \%$ confidence intervals.

Study approval. Animal protocols were approved by the Institutional Animal Care and Use Committee at Duke University.

\section{Author contributions}

$\mathrm{HZ}$ designed research studies, conducted experiments, analyzed data, and wrote the manuscript. QW designed research studies, conducted experiments, and analyzed data. HT, VP, SP, and RP conducted experiments. YJT, ER, MA, and JW made intellectual contributions to studies. BA designed research studies.

\section{Acknowledgments}

Research reported in this publication was supported by the National Institute of Arthritis and Musculoskeletal and Skin Diseases of the National Institutes of Health under award R01AR066765. The content is solely the responsibility of the authors and does not necessarily represent the official views of the National Institutes of Health.

Address correspondence to: Benjamin A. Alman, Duke Hospital South, 200 Trent Drive, Orange Zone 5th floor, DUMC 2888, Durham, North Carolina 27710. Phone: 919.613.6935; Email: ben.alman@duke.edu.

1. Qasem SA, DeYoung BR. Cartilage-forming tumors. Semin Diagn Pathol. 2014;31(1):10-20.

2. Bovée JV, Hogendoorn PC, Wunder JS, Alman BA. Cartilage tumours and bone development: molecular pathology and possible therapeutic targets. Nat Rev Cancer. 2010;10(7):481-488.

3. WHO Classification of Tumours of Soft Tissue and Bone, Vol. 5. In: CDM Fletcher., et al., eds. WHO Classification of Tumours. Ed. 4. Lyon, France: IARC; 2013.

4. Suijker J, et al. Inhibition of mutant IDH1 decreases D-2-HG levels without affecting tumorigenic properties of chondrosarcoma cell lines. Oncotarget. 2015;6(14):12505-12519.

5. Leddy, LR, Holmes RE. Chondrosarcoma of bone. In: Peabody T Attar S, eds. Orthopaedic Oncology. Cham, Switzerland: Springer International Publishing Switzerland; 2014:117-130.

6. Amary MF, et al. Ollier disease and Maffucci syndrome are caused by somatic mosaic mutations of IDH1 and IDH2. Nat Genet. 2011;43(12):1262-1265. 
7. Pansuriya TC, et al. Somatic mosaic IDH1 and IDH2 mutations are associated with enchondroma and spindle cell hemangioma in Ollier disease and Maffucci syndrome. Nat Genet. 2011;43(12):1256-1261.

8. Amary MF, et al. IDH1 and IDH2 mutations are frequent events in central chondrosarcoma and central and periosteal chondromas but not in other mesenchymal tumours. J Pathol. 2011;224(3):334-343.

9. Hirata M, et al. Mutant IDH is sufficient to initiate enchondromatosis in mice. Proc Natl Acad Sci USA. 2015;112(9):2829-2834.

10. Mullen PJ, Yu R, Longo J, Archer MC, Penn LZ. The interplay between cell signalling and the mevalonate pathway in cancer. Nat Rev Cancer. 2016;16(11):718-731.

11. Tsushima $\mathrm{H}$, et al. Intracellular biosynthesis of lipids and cholesterol by Scap and Insig in mesenchymal cells regulates long bone growth and chondrocyte homeostasis. Development. 2018;145(13):null.

12. Wu S, De Luca F. Role of cholesterol in the regulation of growth plate chondrogenesis and longitudinal bone growth. $J$ Biol Chem. 2004;279(6):4642-4647.

13. Yamashita A, et al. Statin treatment rescues FGFR3 skeletal dysplasia phenotypes. Nature. 2014;513(7519):507-511.

14. Kuzu OF, Noory MA, Robertson GP. The Role of Cholesterol in Cancer. Cancer Res. 2016;76(8):2063-2070.

15. Sasaki M, et al. IDH1(R132H) mutation increases murine haematopoietic progenitors and alters epigenetics. Nature. 2012;488(7413):656-659.

16. Long F, Zhang XM, Karp S, Yang Y, McMahon AP. Genetic manipulation of hedgehog signaling in the endochondral skeleton reveals a direct role in the regulation of chondrocyte proliferation. Development. 2001;128(24):5099-5108.

17. Osmak M. Statins and cancer: current and future prospects. Cancer Lett. 2012;324(1):1-12.

18. Campbell VT, et al. Hedgehog pathway inhibition in chondrosarcoma using the smoothened inhibitor IPI-926 directly inhibits sarcoma cell growth. Mol Cancer Ther. 2014;13(5):1259-1269.

19. Farnaghi S, Crawford R, Xiao Y, Prasadam I. Cholesterol metabolism in pathogenesis of osteoarthritis disease. Int J Rheum Dis. 2017;20(2):131-140

20. Porstmann T, et al. SREBP activity is regulated by mTORC1 and contributes to Akt-dependent cell growth. Cell Metab. 2008;8(3):224-236.

21. Montero J, et al. Mitochondrial cholesterol contributes to chemotherapy resistance in hepatocellular carcinoma. Cancer Res. 2008;68(13):5246-5256.

22. Warita K, et al. Statin-induced mevalonate pathway inhibition attenuates the growth of mesenchymal-like cancer cells that lack functional E-cadherin mediated cell cohesion. Sci Rep. 2014;4:7593.

23. Shechter I, Dai P, Huo L, Guan G. IDH1 gene transcription is sterol regulated and activated by SREBP-1a and SREBP-2 in human hepatoma HepG2 cells: evidence that IDH1 may regulate lipogenesis in hepatic cells. J Lipid Res. 2003;44(11):2169-2180.

24. Ricoult SJ, Dibble CC, Asara JM, Manning BD. Sterol regulatory element binding protein regulates the expression and metabolic functions of wild-type and oncogenic IDH1. Mol Cell Biol. 2016;36(18):2384-2395.

25. Horton JD, et al. Combined analysis of oligonucleotide microarray data from transgenic and knockout mice identifies direct SREBP target genes. Proc Natl Acad Sci USA. 2003;100(21):12027-12032.

26. Turcan S, et al. IDH1 mutation is sufficient to establish the glioma hypermethylator phenotype. Nature. 2012;483(7390):479-483.

27. Lu C, et al. IDH mutation impairs histone demethylation and results in a block to cell differentiation. Nature. 2012;483(7390):474-478.

28. Cohen AL, Holmen SL, Colman H. IDH1 and IDH2 mutations in gliomas. Curr Neurol Neurosci Rep. 2013;13(5):345.

29. Matsuda M, et al. SREBP cleavage-activating protein (SCAP) is required for increased lipid synthesis in liver induced by cholesterol deprivation and insulin elevation. Genes Dev. 2001;15(10):1206-1216.

30. Macias AT, Banavali NK, MacKerell AD. DNA bending induced by carbocyclic sugar analogs constrained to the north conformation. Biopolymers. 2007;85(5-6):438-449.

31. Shultz LD, et al. Human lymphoid and myeloid cell development in NOD/LtSz-scid IL2R gamma null mice engrafted with mobilized human hemopoietic stem cells. J Immunol. 2005;174(10):6477-6489.

32. Martin M. Cutadapt removes adapter sequences from high-throuput sequencing reads. Embnet.journal. 2011;17(1):10-12.

33. Kersey PJ, et al. Ensembl Genomes: an integrative resource for genome-scale data from non-vertebrate species. Nucleic Acids Res. 2012;40(Database issue):D91-D97.

34. Dobin A, et al. STAR: ultrafast universal RNA-seq aligner. Bioinformatics. 2013;29(1):15-21.

35. Love MI, Huber W, Anders S. Moderated estimation of fold change and dispersion for RNA-seq data with DESeq2. Genome Biol. 2014;15(12):550.

36. Huber W, et al. Orchestrating high-throughput genomic analysis with Bioconductor. Nat Methods. 2015;12(2):115-121.

37. Mootha VK, et al. PGC-1alpha-responsive genes involved in oxidative phosphorylation are coordinately downregulated in human diabetes. Nat Genet. 2003;34(3):267-273. 